\title{
Internal Structure of Nanoparticle Dimers Linked by DNA
}

\author{
Cheng Chi, ${ }^{\dagger}$ Fernando Vargas-Lara, ${ }^{\ddagger}$ Alexei V. Tkachenko, ${ }^{\dagger}$ Francis W. Starr, ${ }^{\ddagger, *}$ and Oleg Gang ${ }^{\dagger, *}$ \\ ${ }^{\dagger}$ Center for Functional Nanomaterials, Brookhaven National Laboratory, Upton, New York 11973, United States, and ${ }^{\ddagger}$ Physics Department, Wesleyan University, \\ Middletown, Connecticut 06459, United States
}

$\mathrm{T}$ he use of DNA to link nanoparticles (NPs) into complex self-assembled structures is an increasingly popular approach to the bottom-up design of nanoclusters and materials. ${ }^{1-5}$ The selectivity and reversibility of DNA base-pair recognition, coupled with the relative stiffness of double-stranded DNA (dsDNA) ${ }^{6-8}$ and a tunable assembly kinetics, ${ }^{9}$ make DNA an ideal choice to create programmable interactions between NPs. This is accomplished by tethering multiple strands of DNA to a core NP (gold, silver, or CdSe core). ${ }^{10-12}$ The outermost part of tethered strands is singlestranded DNA (ssDNA) with a specific sequence that will either link directly to another NP or connect to another NP via an additional linking strand. ${ }^{13,14}$ The hybridization of ssDNA in linking regions directs the self-assembly of nanoparticles into largerscale structures. Their organization is controlled by the DNA sequences and linker architecture, ${ }^{15-19}$ as well as by the NP geometry. ${ }^{20}$ Using this approach, the precise fabrication of small nanoclusters and the formation of two- and three-dimensional superlattices has been achieved..$^{20-27}$

While the approach of single-step, direct assembly is promising, many biological materials — such as bone, hair, skin, and spider silk ${ }^{28}$ _take advantage of a more complex hierarchical scheme. In such a scenario, assembly at each scale results in units or structures that enable assembly at a larger scale. These processes have been evolved over aeons, so the development of synthetic multiscale self-assembly will prove challenging. In the context of DNA-tethered NPs, the most basic unit in such a hierarchical approach is a dimer of two NPs. As a first step toward a synthetic multiscale assembly, here we examine in detail the structure of dimer units. The structure of these dimers is complicated by the fact that the surface curvature of the NP is on a scale comparable to the length of the connecting DNA. In such
ABSTRACT We construct nanoparticle dimers linked by DNA. These dimers are basic units in a possible multiscale, hierarchical assembly and serve as a model system to understand DNA-mediated interactions, especially in the nontrivial regime when the nanoparticle and DNA are comparable in their sizes. We exam-

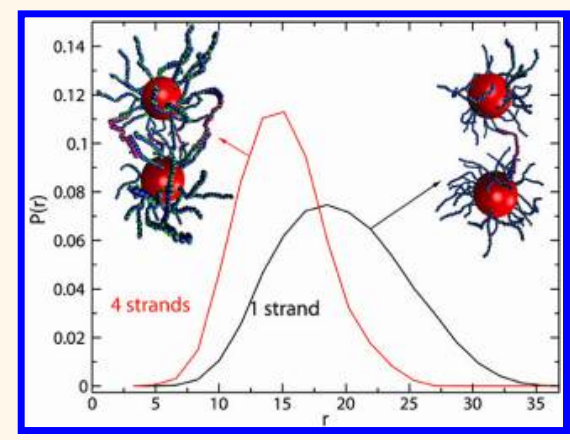
ine the structure of nanoparticle dimers in detail by a combination of scattering experiments and molecular simulations. We find that, for a given DNA length, the interparticle separation within the dimer is controlled primarily by the number of linking DNA. We summarize our findings in a simple model that captures the interplay of the number of DNA bridges, their length, the particle's curvature, and the excluded volume effects. We demonstrate the applicability of the model to our results, without any free parameters. As a consequence, the increase of dimer separation with increasing temperature can be understood as a result of changing the number of connecting DNA.

KEYWORDS: DNA · nanoparticle · self-assembly · SAXS · cluster

a regime, the behavior of polymer chains (such as DNA) attached to the surface is known to deviate significantly from the free-chain behavior. ${ }^{29,30}$

In this paper, we examine the structure of DNA-linked NP dimers by a combination of in situ dynamic light scattering (DLS) and small-angle X-ray scattering (SAXS), complemented by molecular simulations and theory. The DLS method probes a hydrodynamic measure of an angular-averaged dimer size. The in situ SAXS experiments reveal more detailed information on the interparticle distances within the dimer. The numerical modeling provides a detailed molecular picture that helps to interpret the experimental findings and develop a theoretical description.

When the NPs are linked by dsDNA with a separation less than the persistence length, the interparticle distance is a linear function of the DNA length, due to the rigidity of dsDNA. For longer ssDNA linkers, the

\section{* Address correspondence to ogang@bnl.gov, fstarr@wesleyan.edu.}

Received for review April 6, 2012 and accepted July 13, 2012.

Published online July 13, 2012 10.1021/nn301528h

(c) 2012 American Chemical Society 
deviations from linear dependence are well described by the worm-like chain (WLC) model. When the linkage includes a section of SsDNA-which has an order of magnitude smaller persistence length than dsDNA ${ }^{31-35}$ - the flexibility of ssDNA introduces significant complications to understand the dimer separation, especially if the NPs are linked by strands formed by a combination of ssDNA and dsDNA regions. Previous work on NP superlattices with ssDNA linkage ${ }^{14}$ has shown that the interparticle distance can be used to estimate the end-to-end distance of the DNA chains using the WLC model and assuming the persistence length of ssDNA. As we show below, the interparticle distances of the dimer units are significantly smaller than in the NP superlattice for the same length ssDNA linkers, and using a naive WLC description would require an unreasonable persistence length. The molecular simulations reveal that the origin of this discrepancy is in the geometry of NP links. We use this information to construct a simple analytical model that captures the observed behavior. Our findings demonstrate that interparticle separation is dictated by endto-end distance of DNA bridges as well as by the interplay of a particle's curvature and the excluded volume effects.

\section{EXPERIMENTAL DESIGN}

The systems of dimers were prepared using a stepwise surface assembly method (Figure 1), ${ }^{22}$ in which nanoparticle dimers were assembled in a sequential manner by a deposition of DNA-encoded particles on a layer of either surface-grafted ssDNA or DNA-functionalized particles with complementary recognition, as described below. The micrometer-sized (1-4 $\mu \mathrm{m})$ streptavidin-coated magnetic beads ${ }^{22,36}$ were used as surfaces for particle bindings and dimer assembly. The fabricated dimers were composed of nanoparticles with an $11 \mathrm{~nm}$ gold core (AuNP) covered by a shell of ssDNA. The particles within a dimer were connected by ssDNA linkers with ends that are complementary to the ssDNA on the particle shells. The dimers fabrication procedure is schematically illustrated in Figure 1: the first particle $\left(A u \_1\right)$, linker DNAs (DNA_L), and the second particles (Au_2) were added step-by-step to a DNA-grafted surface. The assembled dimers were released from the surface by adding strands with a higher affinity to the surface DNAs, as described in the Methods section.

To study the dependence of the particle-particle separation within the dimer as a function of linker length $L$, we used a series of single-stranded linkers DNA_L (Figure 1, pink strand) as interparticle connectors, where $L$ denotes a number of polythymine bases $(T)$ in a single-stranded linker, not counting recognition ends. We considered $L=0,24,42,60$, or 75 bases. The ratio of pairs-to-DNA linkers corresponds to the average number of linkages within a dimer, equal to 4 in

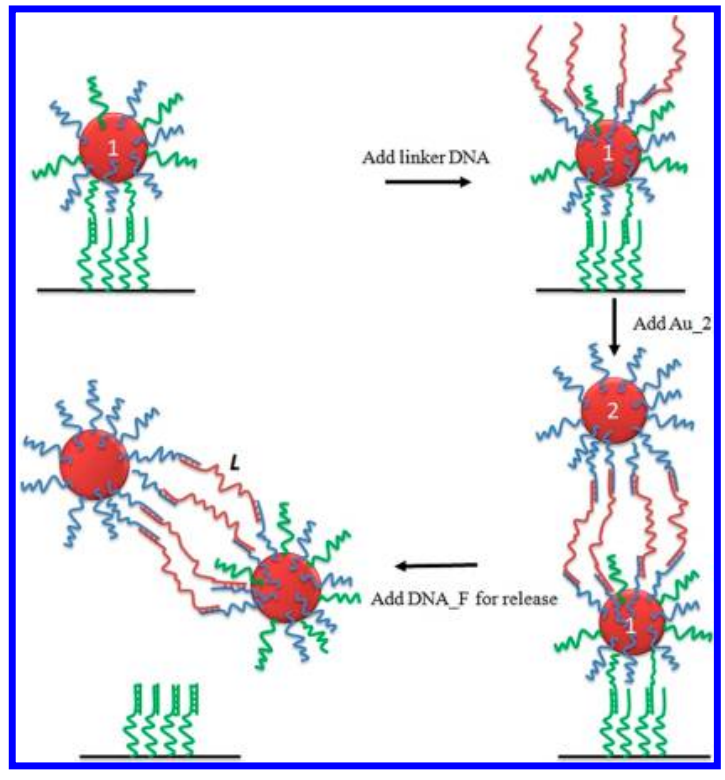

Figure 1. Schematic of the experimental dimer fabrication process using a stepwise surface-encoded method as described in the text and illustration of NPs connected by multiple DNA linkers with length $L$.

this study. Our previous work demonstrated that, at this ratio, a surface efficiently blocks an attachment of linkers to a particle's hemisphere that faces a surface. ${ }^{22}$ Therefore, most of the interparticle connections are established on the top hemisphere of $\mathrm{Au}_{-}$1, opposite to the surface, allowing for hybridization with DNA_2 located on a lower hemisphere of $\mathrm{Au}$ _2. The rigidity of interparticle linkers can be controlled by adding the corresponding complementary strand. In this case, a flexible single-stranded linker becomes a rigid doublestrand connector due to the large increase in persistence length of dsDNA relative to ssDNA.

The DLS measurements conducted during the dimer fabrication process indicated a considerable increase of a number-averaged hydrodynamic diameter $\left(D_{\mathrm{h}}\right)$ of nanoclusters, from $20 \mathrm{~nm}$ for the isolated DNA-coated nanoparticle to about 29 and $37 \mathrm{~nm}$ for dimers with $L$ increasing from 0 to 75 bases, respectively (Figure 2a). The detailed studies, discussed in the Results section, relate the $D_{\mathrm{h}}$ increase with a linker length change. We also examined the population of the fabricated clusters, as dried from the solution, by transmission electron microscopy (TEM). We observed that, for the shortest linker $L=0$, the assembled population consists of about $70 \%$ dimers, about $25 \%$ of unreacted particles, and $5 \%$ multimers $(n \geq 3)$, which is in agreement with our previous studies. For the longest linker, $L=75$, the dimer assembly yield decreased to about $50 \%$ due to the reduction of DNA binding efficiency for longer linkers (Supporting Information Figures S1 and S2). We further applied synchrotron-based SAXS methods to investigate the dependence of the interparticle distance in dimers on $L$, as well as on temperature, by probing a structure factor $S(q)$ of assembled clusters in a solution. 


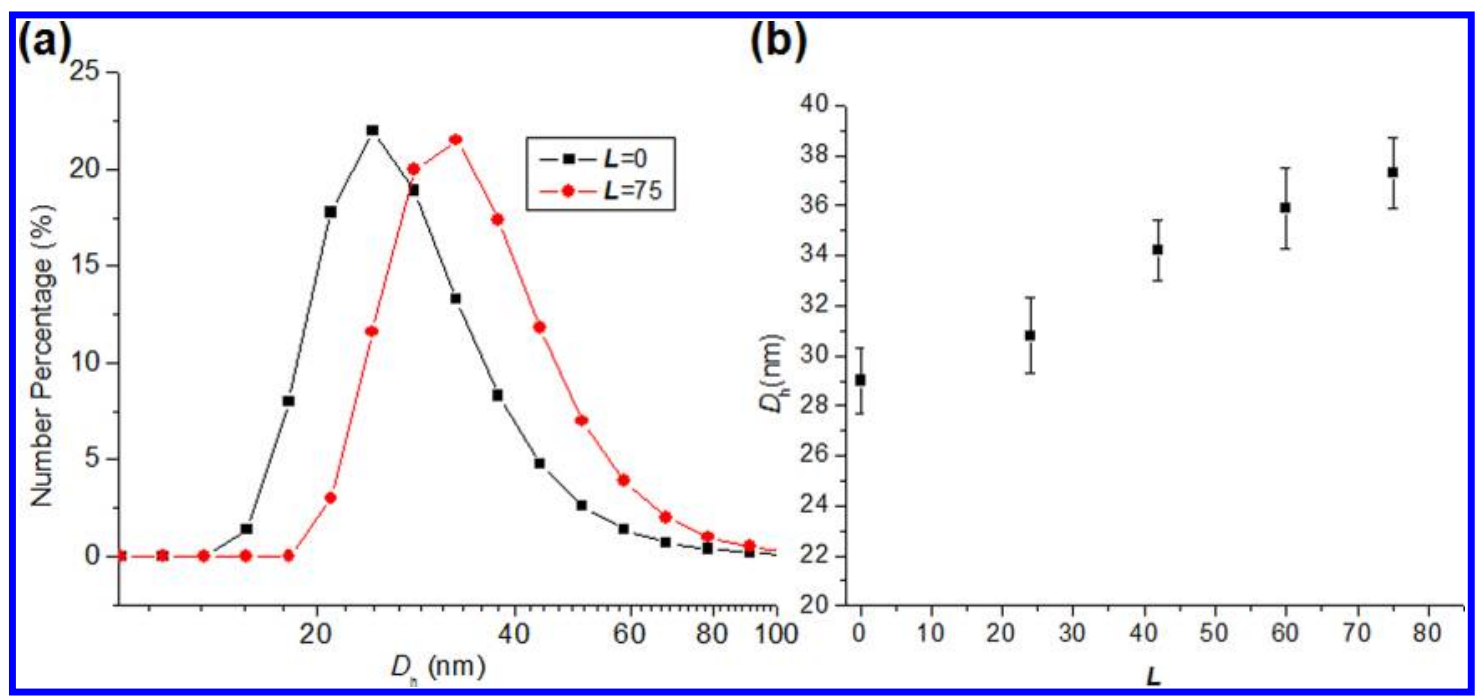

Figure 2. (a) Representative DLS profiles for the solutions of dimers linked by $L=0$ and 75 bases. The number-averaged hydrodynamic diameter, $D_{\mathrm{h}}$, is obtained using a Malvern Zetasizer ZS instrument analysis software. (b) Dependence of $D_{\mathrm{h}}$ on $L$ at room temperature; the error bars are estimated from multiple measurements.

\section{COMPUTATIONAL MODELING}

To aid in interpreting the experimental findings, we use a coarse-grained molecular model that mimics the experimental system. The model enables us to examine the molecular detail of the NPs connected by various numbers of DNA strands as a function of the temperature. In this coarse-grained model, each DNA nucleotide consists of two force sites: one represents the sugarphosphate backbone of a DNA nucleotide, and one carries the $A / C / G / T$ identity of the nitrogenous base (sticky site) (Figure 3). The model has been studied for a variety of systems, and details of the DNA potential can be found in refs $18,19,37$, and 38 , where it has been shown that the model captures the salient features of DNA-driven assembly. The core NP is represented by a single spherically symmetric particle (red spheres in Figure 3). Here, each NP has a diameter $d=14.80 \sigma$, where $\sigma$ is the size of one nucleotide; distance can be mapped to real units by $\sigma \approx$ $0.65 \mathrm{~nm}$ (the typical spacing of ssDNA), yielding a NP diameter $d \approx 10 \mathrm{~nm}$, comparable to the experiments. However, we do not consider the model quantitatively predictive. Each NP has 30 ssDNA attached on its surface. Each ssDNA attached to the NP surface is formed by 16 bases. The outer 6 bases of each chain can hybridize with the linking ssDNA, which is of variable length.

In order to connect the dimers, we add free ssDNA (pink strands of Figure 3) which, like in the experiments, consist of two regions: (i) a central region of variable length $L$ and (ii) ends that are complementary to the ssDNA attached to the NP. The linking region is 6 bases on each end, just like in the ssDNA attached to the NP. To mimic the experimental protocol in which only one hemisphere of the NP has linkers attached, we follow the procedure described in Figure 3. This process can be summarized as follows: two DNA-coated NPs and four linkers (pink strands of Figure 3a) are located at random positions. We randomly select four strands of each facing NP-NP hemisphere (Figure 3b) and hybridize the linkers with the chosen strands (Figure 3c,d). Once the hybridized dimer has formed, we allow the system to equilibrate and evolve without interference. We consider an ensemble of 50 choices for the linkages to evaluate average separations and end-to-end distances.

\section{RESULTS}

Fully Bonded Dimers. We first examine the dependence of the dimer separation on the length $L$ of the DNA linker using the DLS method at $26^{\circ} \mathrm{C}$, where DNA strands should be fully linked. The number-averaged hydrodynamic diameter $\left(D_{\mathrm{h}}\right)$ corresponds to an average cluster size in the population, and for the given particle system, it is indicative of the interparticle distances. We show the distributions of $D_{\mathrm{h}}$ for representative lengths $L=0$ and $L=75$ bases in Figure 2a, which demonstrates a relatively homogeneous cluster population without the presence of large-scale aggregates. From the mean of these distributions, we probe the relationship between the average $D_{\mathrm{h}}$ and $L$, as shown in Figure $2 \mathrm{~b}$. We observe a monotonic, nearly linear growth of $D_{\mathrm{h}}$ from 29 to $37 \mathrm{~nm}$ with increasing $L$ from 0 to 75 bases. The DLS measurement probes an average hydrodynamic diameter of a dimer, which we can relate to the interparticle distance within a dimer using a model in which a dimer is approximated by an ellipsoidal object. ${ }^{22}$ In this model, the shortest axis of the ellipsoid corresponds to the diameter of the particle combined with the ssDNA shell; the DLS measurements show that this axis has a value of $20 \mathrm{~nm}$, independent of $L$. The longest axis relates to the sum of the particle diameters and the DNA linker. The model relates the observed change of $D_{\mathrm{h}}$ from 29 to $37 \mathrm{~nm}$, with an increase of interparticle distance (along 


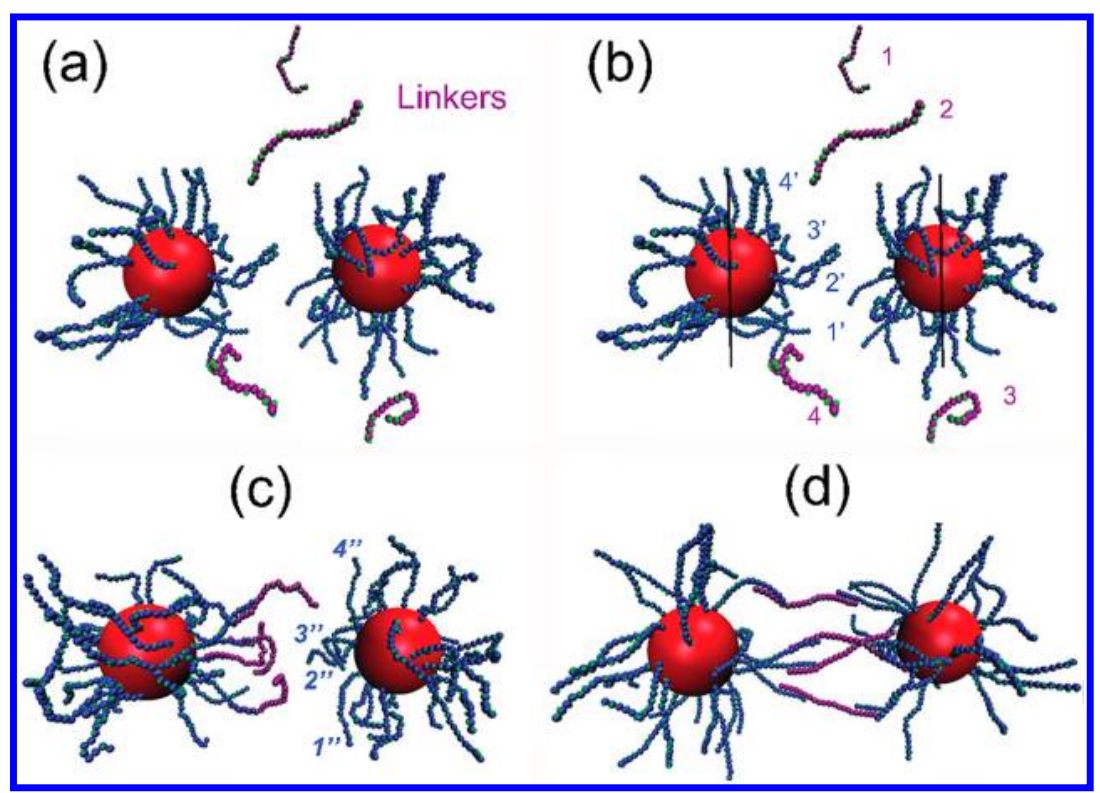

Figure 3. Simulation protocol for dimer formation of two DNA-coated NPs connected by four linkers. (a) Two DNA-coated NPs and four linkers (pink strands) are located at random positions. (b) Four strands of one hemisphere of each NP are randomly selected. (c) Hybridization occurs between the linkers and strands of the left NP. (d) Hybridization with second NP followed by equilibration.

the longer axis of the ellipsoid) from about 17 to $23 \mathrm{~nm}$ for Au particles with a core of $11 \mathrm{~nm}$ and a DNA shell thickness of about $7 \mathrm{~nm}$. We obtain further internal structural details from SAXS measurements.

The in situ SAXS method provides a direct probe of the internal cluster structure and allows for the determination of interparticle distances of dimers with subnanometer precision. The typical 2D scattering patterns from the dimer samples exhibit a faded ring (Figure $4 a, b)$. We show the 1D scattering intensity profiles $I(q)$ obtained from circularly averaging the $2 \mathrm{D}$ scattering patterns and the corresponding structure factors $S(q)$ for studied systems in Figure $4 c, d$, respectively. The first $S(q)$ peak monotonically shifts toward smaller wavevector values, $q$, from 0.0276 to $0.0232 \AA^{-1}$ for a corresponding increase of $L$ from 0 to 75 bases, as determined from a Lorentzian fit. This clearly indicates an increase of the interparticle separation with increasing $L$. The $S(q)$ profiles of dimer clusters in the dilute solution can be described by a dumbbell model $^{39}$

$$
S(q, D)=\frac{\sin (q D)}{2 q D}+\frac{1}{2}
$$

where $D$ is the center-to-center nanoparticle distance. The surface-to-surface distance $r \approx D-d$, where $d=$ $11 \mathrm{~nm}$ is the diameter of the NP gold core.

Figure 5 shows the dependence of $r$ on $L$ for dimers linked with flexible ssDNA or rigid dsDNA connectors and compares the $r$ values with those measured for ordered superlattices in which nanoparticles were linked with ssDNA motifs. For dsDNA connectors, we see an approximately linear growth of the interparticle separation $r$ with increasing $L$. While this behavior is expected when the dsDNA length is sufficiently less than the persistence length of dsDNA $\left(I_{\mathrm{p}} \approx 50 \mathrm{~nm}\right){ }^{40}$ our results indicate a linear regime even when the two lengths are comparable. This can be attributed to the DNA alignment due to multilinker interactions. The observed slope corresponds to $0.24 \mathrm{~nm}$ per base pair, which is consistent with the previously reported SAXS measurements of nanoclusters and 3D systems. ${ }^{13}$ In contrast, the dimers assembled with ssDNA show much weaker dependence of the interparticle separation on the linker length due the greater linker flexibility. More puzzling is the observation that $r$ is smaller (for the same $L$ ) for the case of the dimers connected by ssDNA than for the superlattice system. We note that this deviation cannot be attributed to the difference in the salt concentration between those systems: $0.3 \mathrm{M}$ for superlattice and $0.1 \mathrm{M}$ for dimers. In fact, the reduced salt concentration for dimer systems relative to 3D assemblies should increase (rather than decrease) the persistence length of ssDNA and, correspondingly, increase the NP separations.

To understand the origin of the difference in separation of the dimers as compared to the superlattices, we consider the possibility that the geometry of linkages between NPs may differ between the dimers and the superlattice. To do so, we utilize our simulations to study how the interparticle distance changes when the NPs are connected by exactly one or four linkers. Figure 6 shows the probability distribution function (PDF) of the surface-to-surface distance $r$ of a dimer linked by one or four chains with $L=10$ bases for the linker. The PDF (Figure 6) shows that $r$ is smaller (on average) when the NPs are connected by more than one linker. If all links were parallel to the dimer axis, we would expect little difference between the 


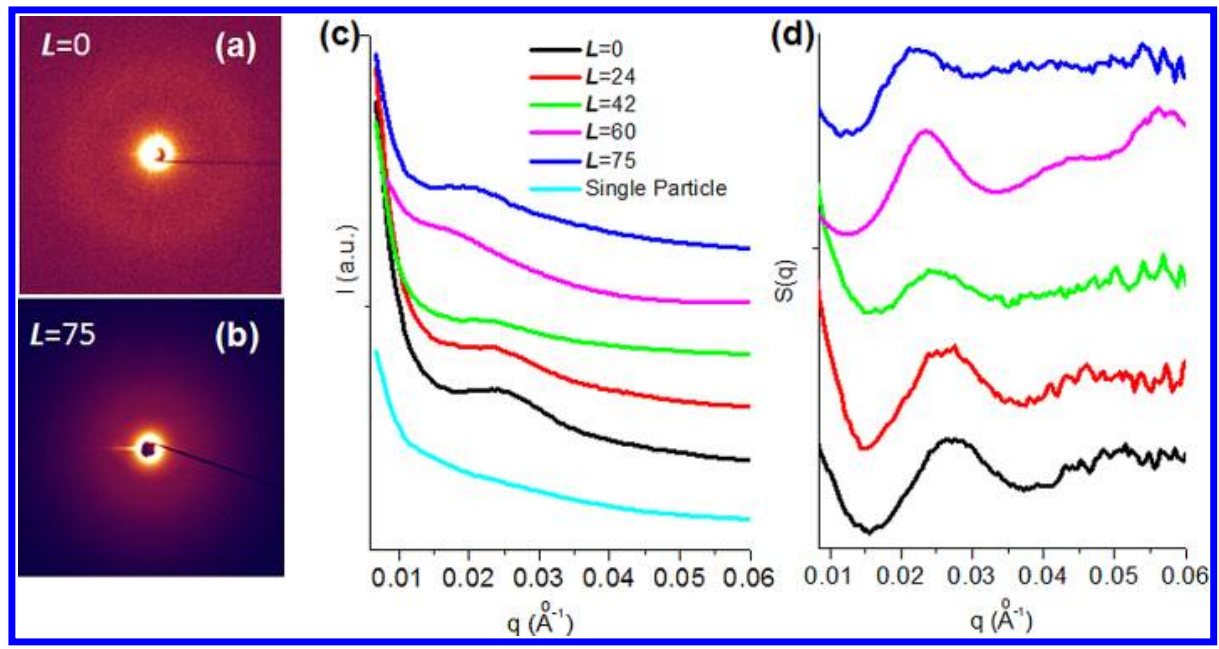

Figure 4. (a,b) Representative 2D small-angle X-ray scattering patterns of dimers for $L=0$ (a) and $L=75$ (b). (c) Relative SAXS intensity, $I(q)$, of dimer systems, as labeled in the inset. (d) Structure factors $S(q)$, obtained as discussed in the method, for systems with corresponding colors shown in (c).

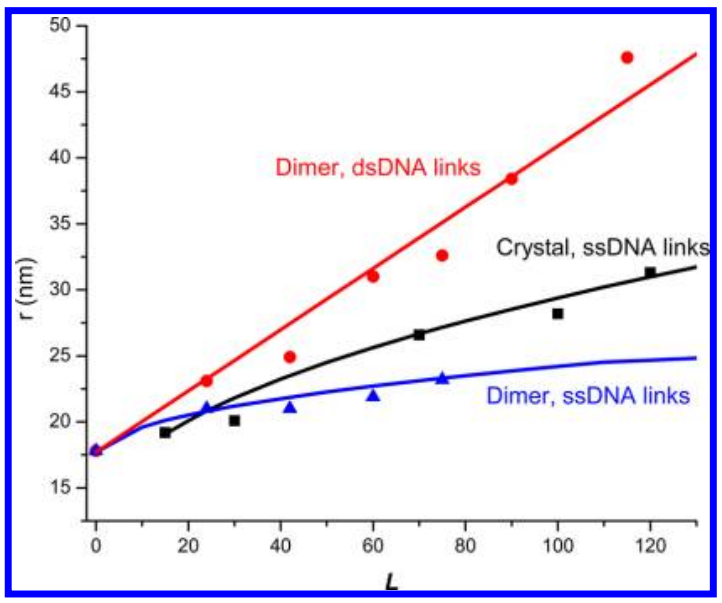

Figure 5. Surface-to-surface distance $r$ as a function of linker length $L$. Red, blue, and black, respectively, correspond to dimers linked by dsDNA, dimers linked by ssDNA, and crystals linked by ssDNA. The symbols are data from the SAXS experiments. For dsDNA-linked dimers, there is a linear relation between $r$ and $L$. For the ssDNA-linked systems, the curves are from fits to the worm-chain model (eq 2), which yields for the crystal $I_{\mathrm{p}}=2.1 \mathrm{~nm}$ and for the dimer $I_{\mathrm{p}}=0.33 \mathrm{~nm}$.

separation of 1 versus 4 linkers. That $r$ is typically smaller with 4 linkers indicates that the geometry of links is more complex. If links cross each other, or occur closer to the poles, this will constrain the overall separation. The illustration of Figure 6 (inset) compares typical geometries for 1 or 4 links, and Figure $\mathrm{S} 6$ contrasts short versus long separation for 4 links, demonstrating that separation is constrained by non-axial links.

In short, the surface-to-surface distance is restricted by the linker with the shortest end-to-end distance, which is typically off the dimer axis. This finding can explain the difference between dimer and superlattice separations. For a NP in the body-centered cubic superlattice, the multibody interactions favor configurations where the linkers are distributed on a particle surface

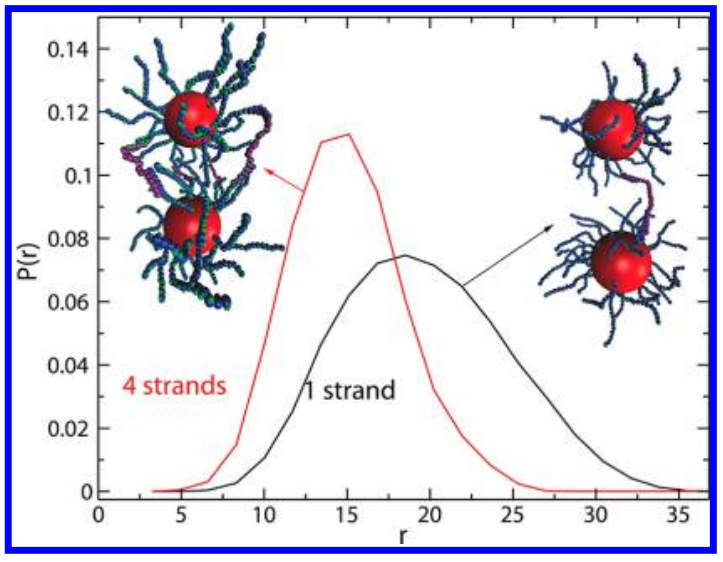

Figure 6. Computed distribution of the surface-to-surface distance $r$ (in a reduced unit, which is base) for a dimer linked by either one or four chains for linker length $L=10$ bases. The NP-NP separation is smaller when more than one linker connects the dimer. The insets show representative dimer configurations at corresponding linker numbers.

that favors particle-to-particle links that are localized in the $\pi / 2$ solid angle between neighboring particles. As a consequence, almost all links lie along the axis of the interparticle separation in the superlattice. Such coaxial links will have a separation that is normally longer than off-axis links.

We next develop a theoretical description of how the separation varies with DNA linkage. Our previous experimental studies indicated that the worm-like chain model (WLC), ${ }^{41}$ in which the mean square endto-end distance of the chain is given by

$$
\left\langle R_{\mathrm{e}}^{2}\right\rangle=2 L \sigma I_{\mathrm{p}}-2 l_{\mathrm{p}}^{2}\left(1-\mathrm{e}^{-L \sigma / I_{\mathrm{p}}}\right)
$$

describes reasonably well the $L$ dependence of the nearest-neighbor interparticle distances of the superlattice for ssDNA linkers with a persistence length $I_{\mathrm{p}}=$ $2.1 \mathrm{~nm}$ (Figure 5). Motivated by this, we test whether our experiments for the dimer systems can be described 


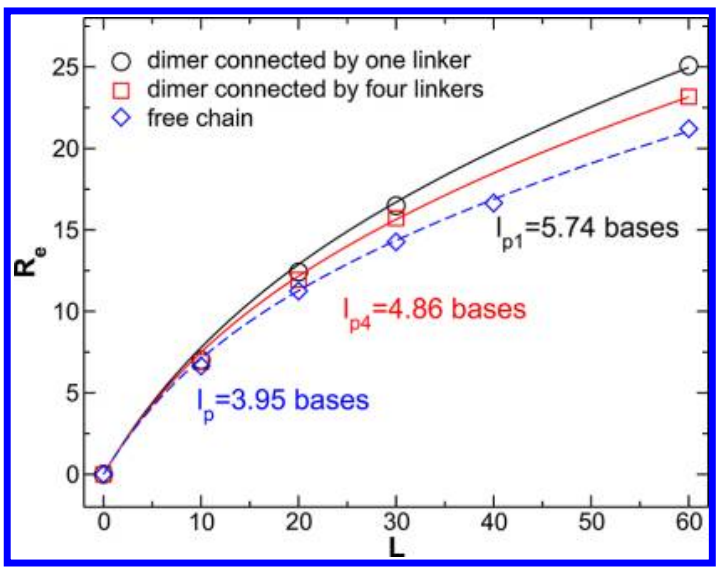

Figure 7. Comparison of the computed end-to-end distance of free ssDNA (blue) in a reduced unit (bases) with the ssDNA connecting the dimer via one linker (black) or four linkers (red). The curves are fits to the worm-chain model (eq 2), which show that the effective persistence length relative to a free chain is larger for NPs connected by one linker and smaller for NPs connected by four linkers.

in the same way. To do so, we need the end-to-end distance of the connecting ssDNA attached to the NP. We can approximate $R_{\mathrm{e}}$ by subtracting from the surfaceto-surface distance $17.8 \mathrm{~nm}$, which is the surface-tosurface distance of dimer linked by $L=0$ connectors obtained from experimental measurement. Using eq 2 , we find a surprisingly small value $I_{\mathrm{p}}=0.33 \mathrm{~nm}$ (about half a base length of ssDNA) for the dimer systems. To rationalize this, we consider that the simulations have shown that the chains can be connected at points far from the axis between particle centers. As a consequence, using the interparticle separation systematically underestimates the end-to-end distances, resulting in an unphysically small value for $I_{\mathrm{p}}$.

We can quantitatively examine how interparticle separation underestimates $R_{\mathrm{e}}$ directly in simulations by comparing $I_{\mathrm{p}}$ evaluated from the actual $R_{\mathrm{e}}$ with that evaluated from the surface-to-surface distance $r$. Following the procedure used to analyze the experiments, we obtain a similarly unreasonably small $I_{p}=0.7$ bases using $r$. In contrast, Figure 7 shows the explicit calculation of $R_{\mathrm{e}}$ for $L=0,10,20,30$, and 60 bases with either one or four linkers connecting NPs; for comparison, we also show a single free chain of ssDNA. The WLC model (eq 2) describes the data well, with a physically reasonable persistence length that is larger than free DNA $\left(I_{\mathrm{p}}=3.95\right.$ bases or $\left.\approx 2.5 \mathrm{~nm}\right)$ for NPs connected by 1 linker ( $I_{\mathrm{p} 1}=5.74$ bases) and by 4 linkers $\left(I_{\mathrm{p} 4}=4.86\right.$ bases $)$. Thus, the effect of being attached between the NPs slightly "stretches" the linking chain. Since we cannot access $R_{\mathrm{e}}$ in experiments and the surface-to-surface separation $r$ used in the WLC model gives unphysical values for $I_{p}$, we need to develop an improved description that can be verified from simulations and applied to the experiments.

We propose a simple theoretical model that accounts for the effects of NPs being linked by a

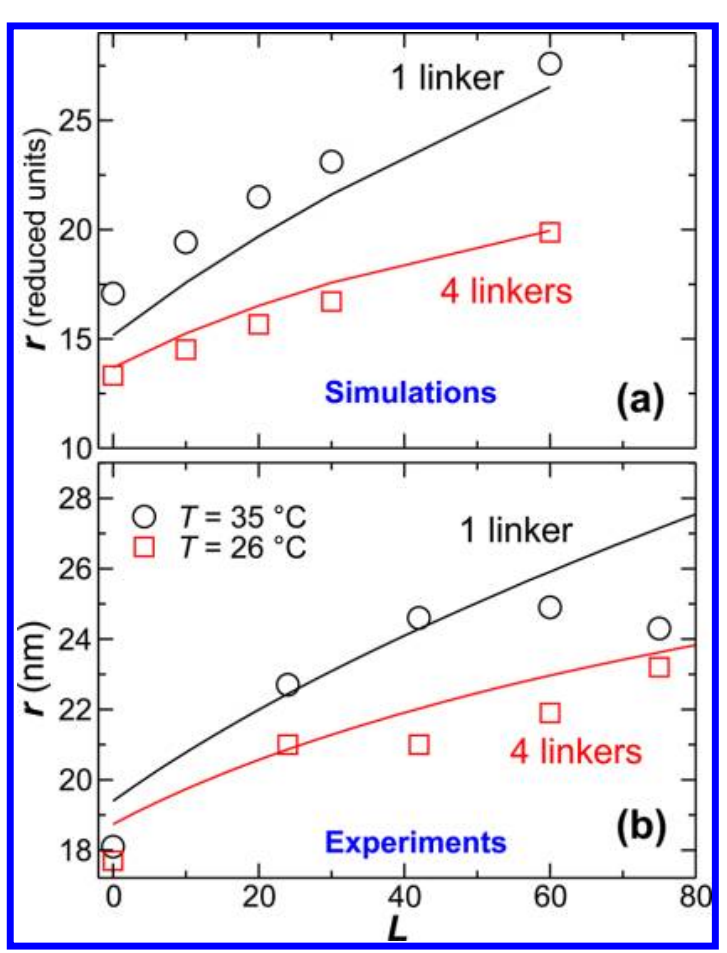

Figure 8. (a) Surface-to-surface separation $r$ as a function of $L$ for the dimer connected via one linker (black) or four linkers (red). The symbols represent the simulation data and the curves the theoretical model proposed in eq 5. (b) Dependence of $r$ on $L$ from experiments at room temperature $\left(26^{\circ} \mathrm{C}\right)$ and before melting $\left(35^{\circ} \mathrm{C}\right)$; solid curves represent the function of $r$ over $L$ estimated from the analytical model with 1 (blue) and 4 (red) linkers in a dimer.

combination of ssDNA and dsDNA regions, as well as multiple linkages. Consider a DNA bridge that connects the two DNA-grafted NPs that is formed by ssDNA regions $\left(L_{\mathrm{ss}}\right.$ bases in total, with $\left.L_{\mathrm{ss}} \sigma>I_{\mathrm{p}}\right)$ and dsDNA regions (each region formed by $d_{i}$ bp, with $d_{i}<I_{\mathrm{p}}{ }^{\mathrm{dsDNA}}$ ). The mean square end-to-end distance $\left\langle R_{\text {bridge }}^{2}\right\rangle$ of such a DNA construct can be estimated by adding the endto-end distances of each individual segment

$$
\left\langle R_{\text {bridge }}^{2}\right\rangle=2 l_{\mathrm{p}} \sigma L_{\mathrm{ss}}+\sigma_{\mathrm{ds}}^{2} \sum_{i} d_{i}^{2}
$$

In this expression, we approximate the ssDNA regions by the Gaussian model (since $L_{s s}>I_{\mathrm{p}}$ ) and the dsDNA regions by the rigid-rod model (since $d_{i}<I_{\mathrm{p}}{ }^{\mathrm{dsDNA}}$ ). Here $\sigma_{\mathrm{ds}} \approx 0.35 \mathrm{~nm}$ is the length per base pair in dsDNA. We can approximate that $\left\langle R_{\text {bridge }}^{2}\right\rangle^{1 / 2} \approx r$, the surface-to-surface separation for a single connecting strand. Figure 8a shows that this simple model roughly describes the linking by a single chain in our numerical simulations. Note that the whole dependence on the linker length, including the initial separation at $L=0$, is calculated without any fitting parameters, assuming the same ssDNA persistence length $I_{\mathrm{p}}=3.95$ bases obtained from the simulations of the free ssDNA.

We next extend this model to account for multiple linkages. When the number of linkers increases, the competition for the binding sites, together with excluded 
volume effects, results in greater distribution of the DNA connections over the NP surface. As shown in the Supporting Information, this effect leads to the following negative correction to the interparticle distance:

$$
\Delta r=-\left(n_{\mathrm{L}}-1\right)\left(\frac{d}{N_{0}}+\frac{I_{\mathrm{p}} \sigma L}{3 \pi d}\right)
$$

Here $n_{\mathrm{L}}$ is the number of linkers in a dimer, and $N_{0}$ is the number of ssDNAs that are bound to a single NP ( 20 for simulations and about 50 in the experiment). By combining eqs 3 and 4 , the resulting expression for the interparticle separation is obtained:

$$
r=\sqrt{2 I_{\mathrm{p}} \sigma L_{\mathrm{ss}}+\sigma_{\mathrm{ds}}{ }^{2} \sum_{i} d_{i}^{2}}-\left(n_{\mathrm{L}}-1\right)\left(\frac{d}{N_{0}}+\frac{I_{\mathrm{p}} L \sigma}{3 \pi d}\right)
$$

The above correction captures the change in interparticle separation due to the multiple linkers observed in simulations (red curve in Figure 8a). Note that all of the parameters are known, so no fit is involved.

A direct application of the same theory to experimental data does not give a satisfactory fit. However, we found that the experimental data for fully linked dimers $\left(n_{\mathrm{L}}=4\right)$ can be successfully described (Figure $8 \mathrm{~b}$ ) after a minor modification of the model:

$$
r=\sqrt{2 I_{\mathrm{p}} \sigma L_{\mathrm{ss}}}+\sigma_{\mathrm{ds}} \sum_{i} d_{i}-\left(n_{\mathrm{L}}-1\right)\left(\frac{d}{N_{0}}+\frac{I_{\mathrm{p}} L \sigma}{3 \pi d}\right)
$$

Here, the first term represents the root-mean-square size of the ssDNA part of the bridge, the second term is the total contour length of the dsDNA segments, and the last term is the multibridge correction (eq 4). Physically, this variation of the model corresponds to the presence of a modest stretching force that is strong enough to orient dsDNA segments but not too strong to significantly stretch the ssDNA fragments of the chain. As a result, dsDNA segments are aligned along the dimer axis, rather than randomly oriented as assumed in eq 5 . The experimental data sufficiently below the melting temperature are well described using this approach for a physically plausible $I_{p}$, from 2 to $2.5 \mathrm{~nm}$. The experimental data to test the effect of the number of linkers will be presented when we discuss the temperature dependence.

The theoretical model (eq 6) also works for more complicated systems, such as dimers connected with combined linkers. In this design, the linker is composed of two strands of ssDNA terminated by mutually complementary 15 base segments (as shown in Figure 9a). This design allows for a longer interparticle separation. We observed that for the $15 \mathrm{bp}$ double-stranded part that is between of the two single-stranded chains, the dimer separation increases by about $5 \mathrm{~nm}$, which is well captured by our theoretical model (Figure 9b).

Temperature Dependence of Dimer Separation. We next examine the temperature $T$ dependence of dimer

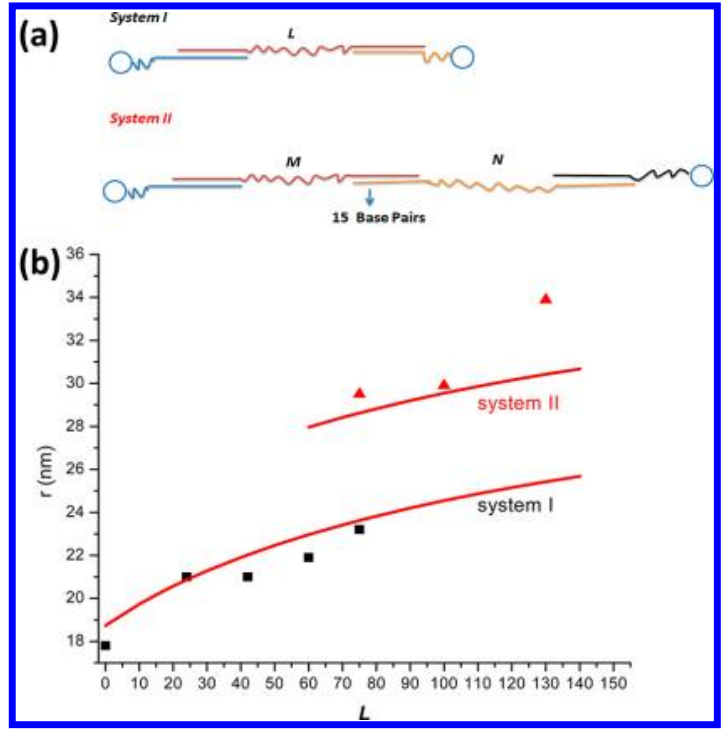

Figure 9. (a) Design of two studied systems: (system I) dimers fabricated using a single-stranded linker, and (system II) dimers fabricated using a combined linker. (b) Surface-to-surface distance $r$ as a function of linker length $L$. For the system II, $L=M+N$. Red curves represent the function of $r$ over $L$ for corresponding systems estimated from theoretical model at $n=4, I_{\mathrm{p}}=2.2 \mathrm{~nm}$.

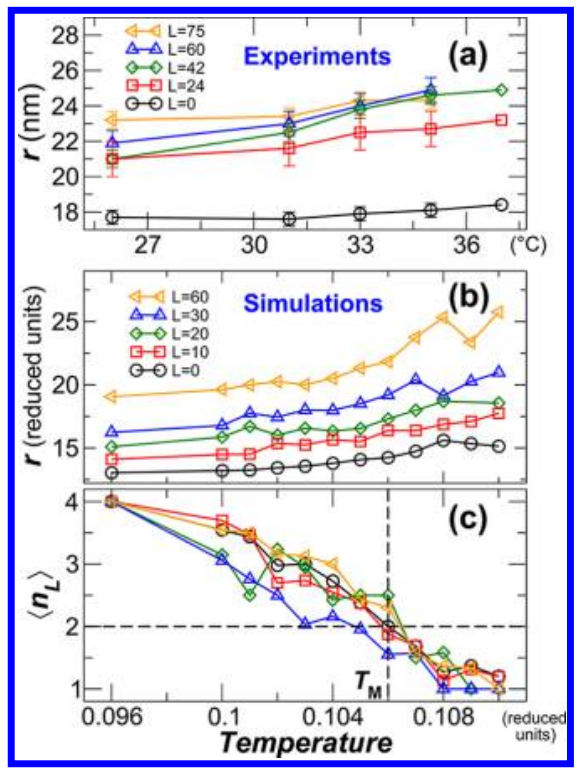

Figure 10. Surface-to-surface separation $r$ as a function of temperature for various $L$ from experiments (a) and simulations (b). The uncertainties of experiments are estimated from multiple measurements and SAXS peak fitting. Like the experiments, the interparticle distance increases with increasing $T$. (c) Average number of linkers $\left\langle n_{\mathrm{L}}\right\rangle$ connecting NP as a function of $T$ from simulations, demonstrating that the change in $r$ is caused by the reducing number of connecting linkers.

separation via the SAXS technique. We perform a gradual heating of the systems from 26 to, 33 to 35 , and 37 and $40^{\circ} \mathrm{C}$. Figure 10a shows the $T$ dependence on $r$ obtained by fitting the data using eq 1 . All systems exhibit a modest increase of $r$ with increasing $T .^{42}$ However, the relatively large error bars do not allow 


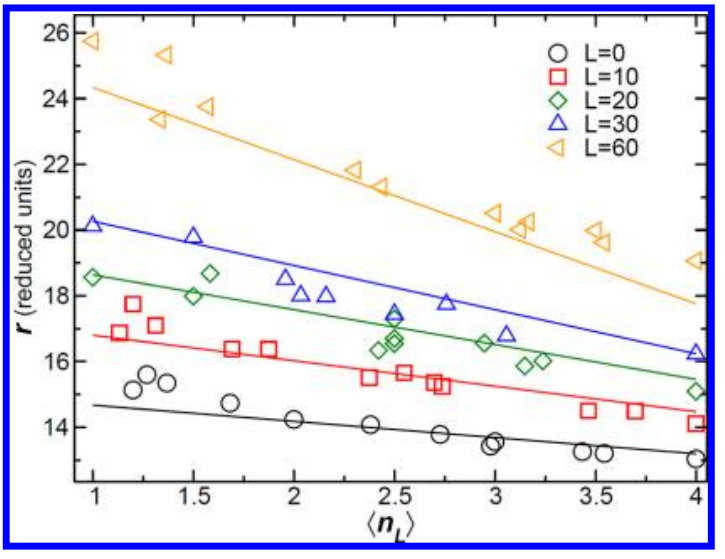

Figure 11. Surface-to-surface separation $r$ as a parametric function of the mean number of links $\left\langle n_{\mathrm{L}}\right\rangle$. The symbols represent the data and the lines the theoretical model proposed in eq 5 .

us to compare a change of separations with temperature as a function of $L$. At the same time, a SAXS monitoring of dimer melting reveals that for shorter linkers melting occurs at a slightly higher $T$, with disassembly of $L=0$ dimers at $40{ }^{\circ} \mathrm{C}$ (Supporting Information Figures S3-S5), which can be attributed to higher local density of DNA. The observed $r$ increase exceeds that expected from the $T$ dependence of the DNA persistence length, which should only change slightly. To understand the origin of the increase of $r(T)$ found experimentally, we examine our molecular simulations.

Following the experimental protocol, in the simulations, we increase the temperature of dimers connected by four linkers from $T=0.096$ (fully connected DNA) to a final temperature $T=0.120$, where the dimers are melted. Figure $10 \mathrm{~b}$ shows $r$ as for this range of $T$. Like the experiments, we find an increase in $r$ when $T$ increases, consistent with Figure 10a. To explain this trend, we directly examine the number of links between the dimers in the simulations. We plot the average number of linkers $\left\langle n_{\mathrm{L}}\right\rangle$ that connect the dimer in the simulations as a function of the temperature in Figure 10c. We find that $\left\langle n_{\mathrm{L}}\right\rangle$ decreases with increasing $T$. As previously shown in Figure 6, when there are more connected linkers, the separation is dominated by the shortest link. Hence, the loss of linkers on increasing $T$ results in increased $r$. This inter-relation is directly seen by plotting parametrically the dimer separation $r$ as a function of $\left\langle n_{\mathrm{L}}\right\rangle$ (Figure 11). In doing so, we eliminate the explicit $T$ dependence of the separation and, consequently, can test the theoretical model proposed in eq 5, generalizing the expression to noninteger values of the number of linkers. Figure 11 shows excellent agreement between the simulation data and the theoretical predictions (without any adjustable parameters). This verifies the predicted dependence on the number of linkers.

Since we now know that increasing temperature has the effect of decreasing the number of links, we can use experimental data from relatively low or high temperature as a way to test the predictions of the model for dimers connected by single or multiple linkers (eq 6), respectively. As shown in Figure 8b, the measured $r(T)$ for all systems is within bounds determined from the analytical model for a single linker connection $(n=1)$ and four linker connections $(n=4)$. Near the melting point, the number of linkers per dimer decreases, and the interparticle separation should approach the $n=1$ value just before dimer melting occurs.

\section{CONCLUSION}

We utilized synchrotron-based small-angle X-ray scattering to characterize the interparticle distance of a dimer of gold nanoparticles linked with DNA chains and interpreted the results with the aid of molecular modeling, leading to the development of an analytical description. We showed that, in the regime when nanoparticles and DNA chains are comparable in size, the separation distance between the two NPs is directly related to the number of links connecting NPs. In the regime when particles and links are comparable in size, the location of links can significantly deviate from the axis between the particles. As a result, the intradimer separation is constrained by off-axis links that yield smaller NP separation than expected for a single chain connecting NPs. We combine these observations in a theoretical model that describes both our numerical and experimental data. The effect of temperature is thus understood as a consequence of changing the mean number of links between NPs. Our findings might play an important role in designing novel materials based on nanoparticles and polymers.

\section{METHODS}

The fabrication procedure of dimers is shown in Figure 1. The first particle, Au_1, was functionalized with a mixture of two types of DNA, denoted as DNA_1 and DNA_1N. These two types of DNA serve the purposes of (i) recognition to surface-grafted DNA (DNA_1NS) and (ii) for the consequent attachment of linkers (DNA_L). Au_1 were deposited on surface functionalized with DNA_1NS due to 15 bases complementary of DNA_1NS and DNA_1N. Following the deposition of the first particle layer, a 4-fold excess of linkers DNA_L relatively to surface-attached particles Au_1 was added in order to hybridize with DNA_1 through 15 complementarity bases. At the third step, particles $\mathrm{Au}$ 2, functionalized with one type of DNA only (denoted as DNA_2), which is complementary to $3^{\prime}$-end of linker DNA_L, were added in a ratio 1:1 relative to Au_1. This resulted in a formation of surface-immobilized dimers due to the hybridization of linkers between $\mathrm{Au}_{-} 1$ and $\mathrm{Au}_{2} 2$ particles which are linked by 4 DNA_L on average (Table 1). Finally, the assembled dimers were released from the surface using a liberating strand (DNA_F) which has a higher affinity to the surface-grafted DNA_1NS than DNA_1N, due to 18 complementarity bases. 
TABLE 1. Single-Stranded DNA Strands Used in This Study

\begin{tabular}{ll}
\multicolumn{1}{c}{ ssDNA } & \multicolumn{1}{c}{ sequence $\left(\mathbf{5}^{\prime}\right.$ ' to $\left.\mathbf{3}^{\prime}\right)$} \\
\hline DNA_1N & ATT GGA TTG GAA GTA TTT TTT TTT TTT TTT-C ${ }_{6} \mathrm{H}_{12}$-SH \\
DNA_1NS & CTT GTG TCT ACT TCC AAT CCA ATT TTT TTT TTT TTT TT-biotin \\
DNA_1 & TTC TCT ACA CTG TCT TTT TTT TTT TTT TTT-C ${ }_{6} \mathrm{H}_{12}$-SH \\
DNA_L & AGA CAG TGT AGA GAA (T)L ATT GTT ATT AGG \\
DNA_2 & HS-C ${ }_{6} \mathrm{H}_{12}$-TTT TTT TTT TTT TTT TAG CCT AAT AAC AAT \\
DNA_F & ATT GGA TTG GAA GTA GAC ACA AG
\end{tabular}

Consequently, particles were dispersed in the 0.1 M PBS (10 nM phosphate buffer, $0.1 \mathrm{M} \mathrm{NaCl}, \mathrm{pH}=7.1$ ).

We applied synchrotron-based SAXS measurements (beamline $X 9$ at the National Synchrotron Light Source) to probe an internal dimer structure. The 2D scattering data were collected with a charge-coupled device area detector (MAR CCD) at wavelength $\lambda=0.8856 \AA$. The SAXS measurements provide information on the structure factor $S(q)$ of clusters in a solution. The 1D intensity profiles, $I(q)$, were obtained by an azimuthally integration of the 2D SAXS pattern. The background-corrected intensity can be represented in a simple form $I(q)=S(q) F(q))$, where $F(q)$ is the form factor of free particles measured from the solution containing the equimolar mixture of free particles. The center-to-center distances between gold nanoparticles within the dimer were obtained from $S(q)$ by accounting for the size of individual AuNPs determined from the separate TEM and SAXS measurements. SAXS measurements were conducted for the samples in $0.1 \mathrm{M}$ PBS buffer placed in a $1 \mathrm{~mm}$ quartz capillary over the temperature range of 26 to $40{ }^{\circ} \mathrm{C}$ with $20 \mathrm{~s}$ exposure time.

Conflict of Interest: The authors declare no competing financial interest.

Acknowledgment. Research was carried out at the Center for Functional Nanomaterials, Brookhaven National Laboratory, which is supported by the U.S. Department of Energy, Office of Basic Energy Sciences, under Contract No. DE-AC0298CH10886. F.V.-L. and F.W.S. thank the NSF for support from Grant No. CNS-0959856.

Supporting Information Available: Detailed information regarding dimer's characterization and theoretical description. This material is available free of charge via the Internet at http:// pubs.acs.org.

\section{REFERENCES AND NOTES}

1. Geerts, N.; Eiser, E. Flying Colloidal Carpets. Soft Matter 2010, 6, 664-669.

2. Geerts, N.; Schmatko, T.; Eiser, E. Clustering versus Percolation in the Assembly of Colloids Coated with Long DNA. Lanamuir 2008, 24, 5118-5123.

3. Geerts, N.; Eiser, E. DNA-Functionalized Colloids: Physical Properties and Applications. Soft Matter 2010, 6, 46474660.

4. Alivisatos, A. P.; Johnsson, K. P.; Peng, X. G.; Wilson, T. E.; Loweth, C. J.; Bruchez, M. P.; Schultz, P. G. Organization of 'Nanocrystal Molecules" Using DNA. Nature 1996, 382, 609-611.

5. Mirkin, C. A.; Letsinger, R. L.; Mucic, R. C.; Storhoff, J. J. A DNA-Based Method for Rationally Assembling Nanoparticles into Macroscopic Materials. Nature 1996, 382, 607609.

6. Seeman, N. C. DNA in a Material World. Nature 2003, 421, 427-431.

7. Niemeyer, C. M. Self-Assembled Nanostructures Based on DNA: Towards the Development of Nanobiotechnology. Curr. Opin. Chem. Biol. 2000, 4, 609-618.

8. Condon, A. Designed DNA Molecules: Principles and Applications. Nat. Rev. Genet. 2006, 565-575.

9. Maye, M. M.; Nykypanchuk, D.; van der Lelie, D.; Gang, O. A Simple Method for Kinetic Control of DNA-Induced
Nanoparticle Assembly. J. Am. Chem. Soc. 2006, 128, 14020-14021.

10. Park, S. Y.; Lytton-Jean, A.; Lee, B.; Weigand, S.; Schatz, G.; Mirkin, C. DNA-Programmable Nanoparticle Crystallization. Nature 2008, 451, 553-556.

11. Hurst, S. J.; Lytton-Jean, A. K. R.; Mirkin, C. A. Maximizing DNA Loading on a Range of Gold Nanoparticle Sizes. Anal. Chem. 2006, 78, 8313-8318.

12. Hill, H. D.; Millstone, J. E.; Banholzer, M. J.; Mirkin, C. A. The Role Radius of Curvature Plays in Thiolated Oligonucleotide Loading on Gold Nanoparticles. ACS Nano 2009, 3, 418-424.

13. Macfarlane, R. J.; Jones, M. R.; Senesi, A. J.; Young, K. L.; Lee, B.; Wu, J. S.; Mirkin, C. A. Establishing the Design Rules for DNA-Mediated Colloidal Crystallization. Angew. Chem., Int. Ed. 2010, 49, 4589-4592.

14. Xiong, H. M.; van der Lelie, D.; Gang, O. Phase Behavior of Nanoparticles Assembled by DNA Linkers. Phys. Rev. Lett. 2009, 102, 015504

15. Hsu, C. W.; Largo, J.; Sciortino, F.; Starr, F. W. Hierarchies of Networked Phases Induced by Multiple Liquid-Liquid Critical Points. Proc. Natl. Acad. Sci. U.S.A. 2008, 105, 13711-13715.

16. Dai, W.; Hsu, C. W.; Sciortino, F.; Starr, F. W. Valency Dependence of Polymorphism and Polyamorphism in DNA-Functionalized Nanoparticles. Langmuir 2009, 26, 3601-3608

17. Dai, W.; Kumar, S. K.; Starr, F. W. Universal Two-Step Crystallization of DNA-Functionalized Nanoparticles. Soft Matter 2010, 6, 6130-6135.

18. Vargas-Lara, F.; Starr, F. W. Stability of DNA-Linked Nanoparticle Crystals I: Effect of Linker Sequence and Length. Soft Matter 2011, 7, 2085-2093.

19. Padovan-Merhar, O.; Vargas-Lara, F.; Starr, F. W. Stability of DNA-Linked Nanoparticle Crystals: Effect of Number of Strands, Core Size, and Rigidity of Strand Attachment. J. Chem. Phys. 2011, 134, 244701.

20. Jones, M. R.; Macfarlane, R. J.; Lee, B.; Zhang, J. A.; Young, K. L.; Senesi, A. J.; Mirkin, C. A. DNA-Nanoparticle Superlattices Formed from Anisotropic Building Blocks. Nat. Mater. 2010, 9, 913-917.

21. Sonnichsen, C.; Reinhard, B. M.; Liphardt, J.; Alivisatos, A.P. A Molecular Ruler Based on Plasmon Coupling of Single Gold and Silver Nanoparticles. Nat. Biotechnol. 2005, 23, 741-745.

22. Maye, M. M.; Nykypanchuk, D.; Cuisinier, M.; van der Lelie, D.; Gang, O. Stepwise Surface Encoding for High-Throughput Assembly of Nanoclusters. Nat. Mater. 2009, 8, 388391.

23. Maye, M. M.; Kumara, M. T.; Nykypanchuk, D.; Sherman, W. B.; Gang, O. Switching Binary States of Nanoparticle Superlattices and Dimer Clusters by DNA Strands. Nat. Nanotechnol. 2010, 5, 116-120.

24. Maye, M. M.; Gang, O.; Cotlet, M. Photoluminescence Enhancement in CdSe/ZnS-DNA Linked-Au Nanoparticle Heterodimers Probed by Single Molecule Spectroscopy. Chem. Commun. 2010, 46, 6111-6113.

25. Xiong, H. M.; Sfeir, M. Y.; Gang, O. Assembly, Structure and Optical Response of Three-Dimensional Dynamically Tunable Multicomponent Superlattices. Nano Lett. 2010, 10, 4456-4462.

26. Macfarlane, R. J.; Lee, B.; Jones, M. R.; Harris, N.; Schatz, G. C.; Mirkin, C. A. Nanoparticle Superlattice Engineering with DNA. Science 2011, 334, 204-208.

27. Crocker, J. Nanomaterials: Golden Handshake. Nature 2008, 451, 528-529.

28. Ritchie, R. O.; Buehler, M. J.; Hansma, P. Plasticity and Toughness in Bone. Phys. Today 2009, 62, 41-47.

29. Nykypanchuk, D.; Maye, M. M.; van der Lelie, D.; Gang, O. DNA-Based Approach for Interparticle Interaction Control. Langmuir 2007, 23, 6305-6314.

30. Milner, S. T. Polymer Brushes. Science 1991, 251, 905-914.

31. Bustamante, C.; Bryant, Z.; Smith, S. B. Ten Years of Tension: Single-Molecule DNA Mechanics. Nature 2003, 421, 423427. 
32. Murphy, M. C.; Rasnik, l.; Cheng, W.; Lohman, T. M.; Ha, T. J. Probing Single-Stranded DNA Conformational Flexibility Using Fluorescence Spectroscopy. Biophys. J. 2004, 86, 2530-2537.

33. Rivetti, C.; Walker, C.; Bustamante, C. Polymer Chain Statistics and Conformational Analysis of DNA Molecules with Bends or Sections of Different Flexibility. J. Mol. Biol. 1998, 280, 41-59.

34. Mastroianni, A. J.; Sivak, D. A.; Geissler, P. L.; Alivisatos, A. P. Probing the Conformational Distributions of Subpersistence Length DNA. Biophys. J. 2009, 97, 1408-1417.

35. Mathew-Fenn, R. S.; Das, R.; Harbury, P. A. B. Remeasuring the Double Helix. Science 2008, 322, 446-449.

36. Chi, C.; Maye, M. M.; Stadler, A. L.; van der Lelie, D.; Gang, O. Sensing Nucleic Acids with Dimer Nanoclusters. Adv. Funct. Mater. 2011, 21, 1051-1057.

37. Largo, J.; Starr, F. W.; Sciortino, F. Self-Assembling DNA Dendrimers: A Numerical Study. Langmuir 2007, 23, 58965905.

38. Starr, F. W.; Sciortino, F. Model for Assembly and Gelation of Four-Armed DNA Dendrimers. J. Phys.: Condens. Matter 2006, 18, L347-L353.

39. Kaya, H. Scattering from Cylinders with Globular End-Caps. J. Appl. Crystallogr. 2004, 37, 223-230.

40. Bustamante, C.; Smith, S. B.; Liphardt, J.; Smith, D. SingleMolecule Studies of DNA Mechanics. Curr. Opin. Struct. Biol. 2000, 10, 279-285.

41. Doi, M.; Edwards, S. The Theory of Polymer Dynamics; Oxford University Press: New York, 1998; p 408.

42. Smith, B. D.; Dave, N.; Huang, P. J. J.; Liu, J. W. Assembly of DNA-Functionalized Gold Nanoparticles with Gaps and Overhangs in Linker DNA. J. Phys. Chem. C 2011, 115, 7851-7857. 